\title{
Flat panel detector-CT with endovenous injection. Description of a novel technique for obtaining cerebral arteries imaging: Technical note
}

\author{
Susana Vega-Montesinos ${ }^{1}$, Marco Zenteno ${ }^{1}$, Carlos Andres Ferreira-Prada ${ }^{1}$, \\ Jorge A. Santos-Franco ${ }^{2}$, Angel Lee ${ }^{3}$, Luis Rafael Moscote Salazar ${ }^{4}$ \\ ${ }^{1}$ Instituto Nacional de Neurologia y Neurocirugia "Manuel Velasco Suarez", Mexico \\ ${ }^{2}$ IMSS "La Raza”, Mexico \\ ${ }^{3}$ Hospital Angeles del Pedregal, Mexico \\ ${ }^{4}$ Universidad de Cartagena, Colombia
}

\section{Introduction}

The diagnosis and management of cerebral vascular disease has improved due to the constant development of imaging techniques. Digital subtraction angiography (DSA) is the gold standard, but still involves high cost and risk (1).

The certainty of the images obtained by noninvasive methods currently has facilitated the identification of therapeutic surgical or endovascular decisions in many centers of the world regardless of the ASD. At other times, the CT angiography is useful as a tool for control and monitoring in patients already treated (2-6), however it is still ineffective for proper evaluation of intra-stent stenosis (7-9).

The flat-panel detector CT (FPDCT) is a relatively new technology that provides extremely useful images in cerebral vascular disease, especially for its ability to show relationships between soft tissue and bone, detect complications during the procedure, display of intra and extracranial coils and stents, however it require the intraarterial injection of contrast material (10-12).

There are few articles that show the intravenous injection technique for FPDCT. $\mathrm{n}$ this paper we show a novel technique developed at the National Institute of Neurology and Neurosurgery for imaging with intravenous injection.

Equipment. We used a biplane system C-arm with flat detector angiography, AXIOM Artis dBA (Siemens, Erlangen, Germany) with a system of dynamic imaging plane detector with high spatial resolution and contrast resolution of 14 bits, detector input plane $30 \times 40(48 \mathrm{~cm}$ diagonal) with a pixel size of $154 \mathrm{um}$ and input level detector 20 x $20(25 \mathrm{~cm}$ diagonal) with a pixel size of $184 \mathrm{um}$, with acquisition of DSA images in real time with up to $7.5 \mathrm{imag} . / \mathrm{s}, 10,15$, January 30 imag. / s in biplane in 1024 x 1024 matrix 
with a depth of up to 14-bit digitization (7.5 imag./s at 2,480 x 1,920 matrix) DR Dynavision rotational angiography and DSA Dynavision up 60 imag./s.

The specifications for the processing of the images were: standardized platform with syngo user interface, simultaneous display of subtraction fluoroscopy/digital subtraction fluoroscopy and original images by Roadmap Plus (digital subtraction fluoroscopy plus); DICOM Print (services printing), DICOM Send/storage commitment (commitment storage/ shipping) and DICOM query/Retrieve (search and retrieval); full DICOM functionality in both, patient data acquisition and in documentation and archiving; and Dyna CT program (Siemens)

Intravenous FD-CTA imaging was performed after removal of the diagnostic catheter and introducer sheath about 25 to $30 \mathrm{~min}$ after the last DSA series. The patient was asked to close the eyes and to breathe calmly. A dedicated FD-CT program (DynaCT, Siemens AG, Healthcare Sector, For- chheim, Germany) with a mask run (native) and a second fill run (contrast-enhanced) rotation as described before [11] with an optimised contrast medium application method was used.

Data acquisition per run was carried out using the following parameters: acquisition time $10 \mathrm{~s}$ per run, $70 \mathrm{kV}, 512 \times 512$ Matrix,

projection on $30 \times 40 \mathrm{~cm}$ flatpanel size, $200^{\circ}$ total angle, $0.8^{\circ} /$ Frame, 250 frames total.
To control contrast medium influx we used the bolus watching method [11] to visualise a proper time point to start the fill run acquisition as follows (Fig. 1): contrast medium injection is started $5 \mathrm{~s}$ after the start of the mask run. The mask run acquisition is completed within $10 \mathrm{~s}$. The C-arm returns to the start position, this requires an interval of $5 \mathrm{~s}$.

It can be assumed that within $10 \mathrm{~s}$ contrast material injected into a peripheral cubital vein will not reach the cerebral arteries. After the $\mathrm{C}$-arm returns to the start position (10 s after contrast medium injection) then standard 2D-DSA acquisitions are initiated at a rate of two images per second. When contrast opacification of the large arteries is visible the second rotation (fill run) is initiated manually.

Acquisition method FPD-CT: iopamidol 300 (0.612 g) (Iopamiron, Bayer Schering, Germany) was injected via, preferably left cubital vein. The injection volume was 60 $\mathrm{cc}$ at a rate of $4 \mathrm{cc} / \mathrm{seg}$, injection pressure 150 psi with a power injector (Mark V ProVis Angiographic Injection System, Medrad). For adquisition we used the program DynaCT (Siemens) with the following parameters: 20 seconds of rotation, 60 frames per second (1200 images) with a projection angle of $219^{\circ}$ CTDIw about $35 \mathrm{mGy}$, which allows the reconstruction of a not truncated volume of approximately $25 \mathrm{~cm}$ (on a diagonal plane) with a pixel size of $184 \mathrm{um}$.

The patient is positioned supine on the angiography table, as usual, upon proper 
head position confirmation by fluoroscopy, is activated the head DynaCT 20s program to immediately start injecting intravenous contrast material. With a delay of 10 seconds starts acquisition of the mask (mask run) that surely leads to the acquisition fill run, without using the display method of the contrast bolus described in elsewhere article (7).

The images were sent to the workstation Leonardo (Siemens). The images obtained by the described technique in this article were compared with DSA images, volumetric reconstructions and of MIP angiotomography. DSA images were obtained in two teams, one Axiom Artis angiography (Siemens) and Sygo angiography (Siemens) and those of angiography by 64-slice CT scanner (Siemens). The images were compared by two independent observers and results were collated later. The image comparison was made in order to assess specific aspects: 1) subjective, such as vascular image quality, image quality of intra-aterial devices such as stents and coils, image quality within stents, quality of close vascular images or embedded within bone structures, and 2) objectives, such as vascular diameter measurement, measurement of intracranial aneurysms, measuring of intra-stent stenosis, relation with bony structures.

\section{Ilustrative case}

Case: Female, 40 years old patient, who after indirect cervical trauma developed severe neck pain radiating to the occipital region. It was suspected arterial dissection. The CT angiography confirmed the suspicion, showing extensive dissection of the left vertebral artery that compromised their V2 and V3 segments. SDA was performed and the same procedure was treated by placing three self-expanding stents of $5 \mathrm{~mm} \times 30 \mathrm{~mm}$ (Wallstent, Boston Scientific) in V2 segment and balloonexpandable stent (Taxus, Boston Scientific) in V3 segment. Control at 6 months was obtained with CT angiography and intravenous DynaCT. (Figure $1 \mathrm{~A}, \mathrm{~B}, \mathrm{C}$, D, E).

\section{Discussion}

The diagnosis and management of cerebral vascular disease has greatly improved thanks to the constant development of imaging techniques. Certainly, cerebral angiography since its inception became the gold standard technique that has been enriched progressively from the digital subtraction images in 3D to arterial injection. However despite this spectacular development remains a high cost procedure that involves economic and risk problems (1). Complications may include cerebral ischemia, dissection, intra or extracranial hematomas typically range from 0.6 to $1 \%$ even in well-equipped centers and in experienced hands. 

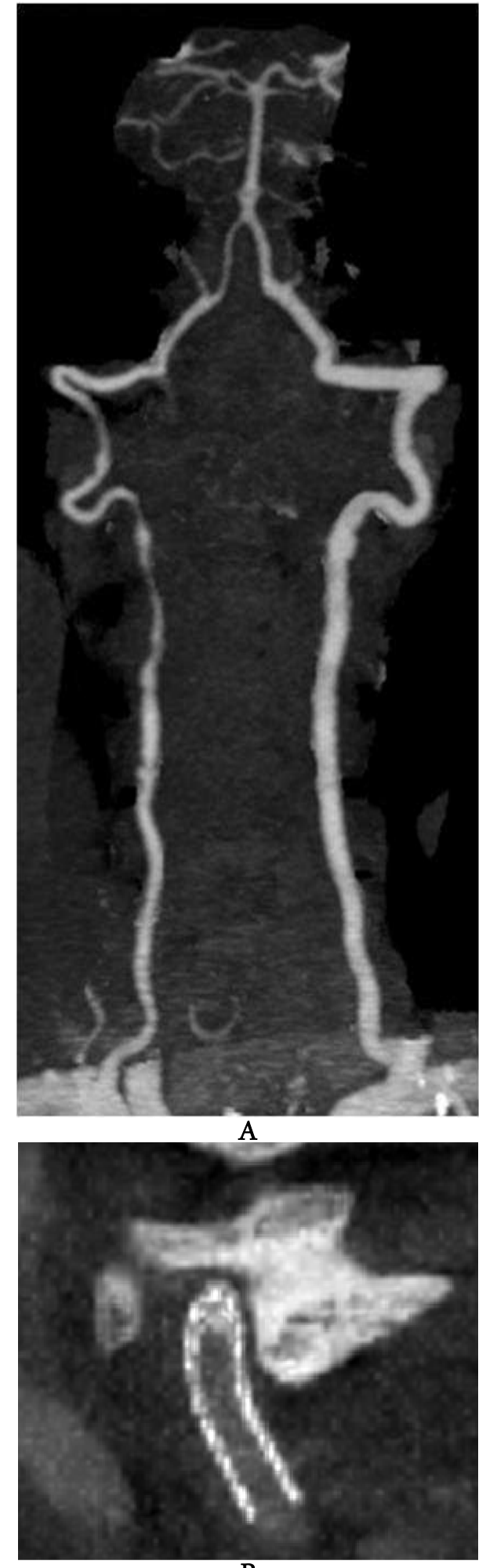

B
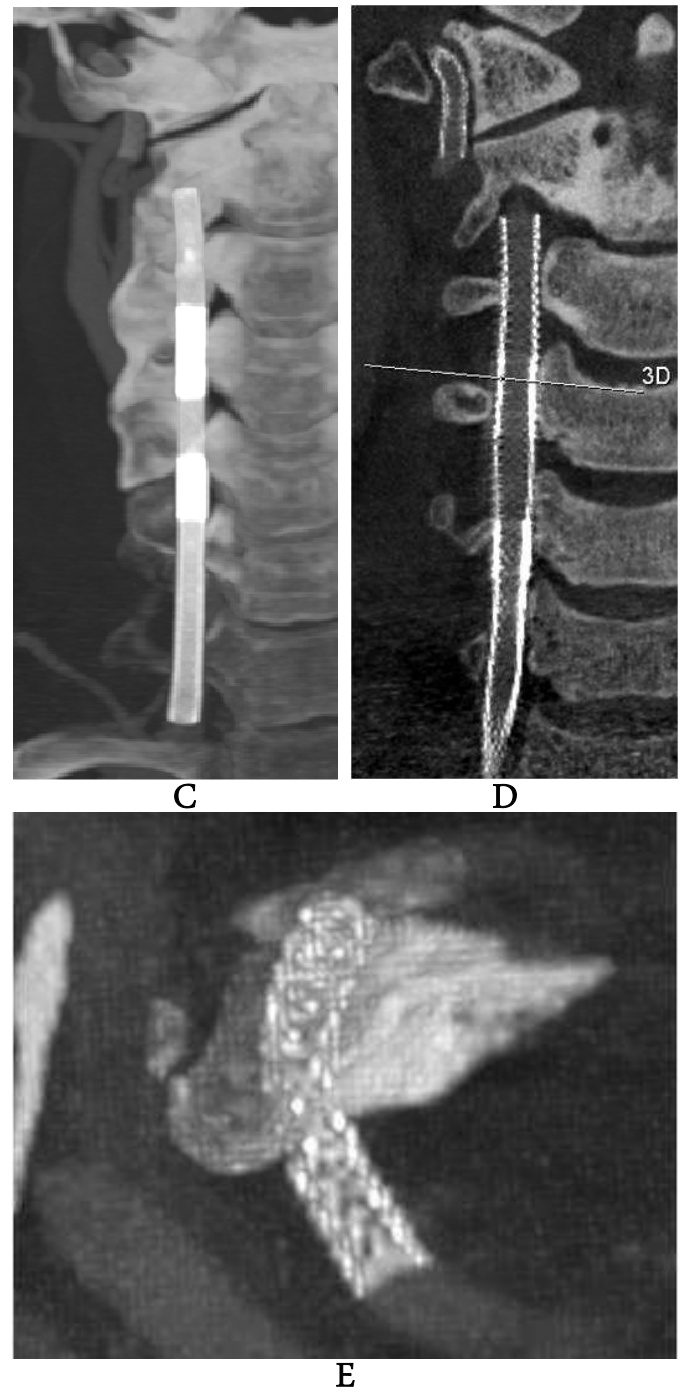

Figure 1

In addition and in parallel, the development of noninvasive methods such as CT and MRI angiography, has led that, on many occasions and in various institutions around the world, the MIP and volumetric reconstructions been taken as the only parameter to therapeutical surgical or endovascular decisions regardless of cerebral angiography. In other circumstances, the CT angiography is useful as a tool for control and 
monitoring in patients already treated (2), as has happened in our experience in the management of intracranial aneurysms (36). In contrast, the MRI angiography is not useful in patients already treated by artifacts that modify endovascular materials (coils and / or stents) (7), and that the image quality is often overcome by angioCT. However, in regard to the evaluation of intrastent stenosis should be noted that the multislice CT scanners have a limited spatial resolution that makes them useless for this purpose (7-9).

The DynaCT is a new technology consisting in the use of a $\mathrm{C}$-arm equipped with flat panel detectors to produce images of CT cone-bean volume, all while the patient is in the angiography table.

It has been shown that the images obtained by DynaCT with arterial contrast injection are useful for understanding and analyzing of cerebral vascular diseases, especially for its ability to show relationships between soft tissue and bone, detect complications during the procedure, display of intra and extracranial coils and stents, however it require the intraarterial injection of contrast material (10-13). These data are not always obtained with digital subtraction angiography, and image quality can be superior in the $3 \mathrm{D}$ angiography, and may be similar to the advantage of CT angiography obtained without moving the patient from the hemodynamics room. However DynaCT technique persist with the inconveniences of all endovascular invasive procedures, as mentioned above (1), while intravenous contrast application avoids the risks associated with intra-arterial catheterization.

In our study the parameters were sufficient to be compatible with the intravenous injection with imaging that we consider to be superior to CT angiography regardless of intraaterial injection of intraraterial DynaCT. This advantage has been extremely useful in our patients, especially those attending outpatient aftercare control.

It should be mentioned that technique used at our institution differs in some respects to that used in other centers. (13). The volume of contrast used is smaller (60cc vs 100cc), not diluted contrast and lower osmolarity 300uo vs. 370 uo. While there seems not to be a remarkable difference, however it is important when is tried the possibility of reducing kidney damage in susceptible patient, e.g., diabetes mellitus, arterial hypertension, etc. It should be noted that this aspect closely resembles the contrast volume used in CT angiography. Regarding the duration of the procedure, in our technique, we obviate the preview contrast injection, which is always imaged in 20 seconds, whereas the previously described technique requires approximately 30 seconds. As mentioned for the contrast, this appears to be a minor daub, however, we believe it is a possible gain to reduce at maximum, the emission and radiation exposure to both, the patient and the personal occupationally exposed, without sacrificing image quality. 


\section{Conclusions}

With the technique of intravenous DynaCT, described in this article, we can obtain good quality images, with less contrast quantity and osmolarity, and less exposure time. This technique is also safe without the complications of intra-arterial injection and without the need to mobilize the patient from the angiography suite. It can also be a valuable tool for both outpatient, initial diagnosis and follow-up. The assessment of intrastent images appear to be more accurate than angiotomography; it is required a larger comparative study that aims to prove statistically this aspect, and also would be avoided the risk inherent in intra-arterial catheterization.

\section{References}

1. Willinsky RA, Taylor SM, TerBrugge K, Farb RI, Tomlinson G, Montanera W. Neurologic complications of cerebral angiography: prospective analysis of 2,899 procedures and review of the literature. Radiology 2003;227:522-8.

2. Buhk J-H, Kallenberg K, Mohr A, Dechent P, Knauth M. Evaluation of angiographic computed tomography in the follow-up after endovascular treatment of cerebral aneurysms--a comparative study with DSA and TOFMRA. Eur Radiol 2009;19:430-6.

3. Zenteno M, Modenesi Freitas JM, Aburto-Murrieta Y, Koppe G, Machado E, Lee A. Balloon-expandable stenting with and without coiling for wide-neck and complex aneurysms. Surg Neurol 2006;66:603-10; discussion 610 .

4. Zenteno MA, Santos-Franco JA, Freitas-Modenesi JM, Gómez C, Murillo-Bonilla L, Aburto-Murrieta Y, Díaz-
Romero R, Nathal E, Gómez-Llata S, Lee A. Use of the sole stenting technique for the management of aneurysms in the posterior circulation in a prospective series of 20 patients. J Neurosurg 2008;108:1104-18.

5. Zenteno M, Gómez CR, Santos-Franco J, Vinuela F, Aburto-Murrieta Y, Lee A. Ten-year follow-up of giant basilar aneurysm treated by sole stenting technique: a case report. J Med Case Rep 2010;4:64.

6. Santos-Franco JA, Zenteno M, Lee A. Dissecting aneurysms of the vertebrobasilar system. A comprehensive review on natural history and treatment options. Neurosurg Rev 2008;31:131-40; discussion 140. 7. Struffert T, Kloska S, Engelhorn T, Deuerling-Zheng Y, Ott S, Doelken M, Saake M, Köhrmann M, Doerfler A. Optimized intravenous Flat Detector CT for noninvasive visualization of intracranial stents: first results. Eur Radiol 2011;21:411-8.

8. Turk AS, Rowley HA, Niemann DB, Fiorella D, Aagaard-Kienitz B, Pulfer K, Strother CM. CT angiographic appearance of in-stent restenosis of intracranial arteries treated with the Wingspan stent. AJNR Am J Neuroradiol 2007;28:1752-4.

9. Trossbach M, Hartmann M, Braun C, Sartor K, Hähnel S. Small vessel stents for intracranial angioplasty: in vitro evaluation of in-stent stenoses using CT angiography. Neuroradiology 2004;46:459-63.

10.Namba K, Niimi Y, Song JK, Berenstein A. Use of Dyna-CT Angiography in Neuroendovascular DecisionMaking. A Case Report. Interv Neuroradiol 2009;15:6772 .

11.Benndorf G, Claus B, Strother CM, Chang L, Klucznik RP. Increased cell opening and prolapse of struts of a neuroform stent in curved vasculature: value of angiographic computed tomography: technical case report. Neurosurgery 2006;58:ONS-E380; discussion ONS-E380.

12.Benndorf G, Strother CM, Claus B, Naeini R, Morsi $\mathrm{H}$, Klucznik R, Mawad ME. Angiographic CT in cerebrovascular stenting. AJNR Am J Neuroradiol 2005;26:1813-8.

13. Heran NS, Song JK, Namba K, Smith W, Niimi Y, Berenstein A. The utility of DynaCT in neuroendovascular procedures. AJNR Am J Neuroradiol 2006;27:330-2. 